Research Article

\title{
Physicochemical Studies on the Interaction between Sodium Dodecyl Sulfate and Methylene Blue in Methanol-Water Mixed Solvent Media
}

\author{
Pawan Shah $(\mathbb{D}$, Nilam Jha $(\mathbb{D}$, and Ajaya Bhattarai $(\mathbb{D}$ \\ Department of Chemistry, M.M.A.M.C., Tribhuvan University, Biratnagar, Nepal \\ Correspondence should be addressed to Ajaya Bhattarai; bkajaya@yahoo.com
}

Received 4 August 2020; Revised 4 November 2020; Accepted 20 November 2020; Published 3 December 2020

Academic Editor: Suresh Kannan Balasingam

Copyright () 2020 Pawan Shah et al. This is an open access article distributed under the Creative Commons Attribution License, which permits unrestricted use, distribution, and reproduction in any medium, provided the original work is properly cited.

\begin{abstract}
The surface activity and the process of micellization of sodium dodecyl sulfate (SDS) with methylene blue (MB) in a methanolwater mixed solvent system were investigated by tensiometry and conductometry at $298.15 \mathrm{~K}$. The measurements of surface tension and conductivity of SDS-MB complex were carried up to 0.3 volume fractions of methanol because the resulting complex appeared turbid above 0.3 volume fractions of methanol. The critical micelle concentration (CMC) of the SDS was determined from both tensiometry and conductometry. The CMC of SDS increased with the increasing volume fraction of methanol due to the inclusion of methanol in the micelles and decreased in the presence of methylene blue due to the reduction of electrostatic repulsion within the anionic moiety of SDS by the positive charge of the added dye. Different properties like surface excess concentration $\left(\Gamma_{\max }\right)$, minimum surface area $\left(A_{\min }\right)$, Gibbs free energy of micellization $\left(\Delta G_{\mathrm{m}}^{o}\right)$, surface pressure $\left(\pi_{\mathrm{CMC}}\right)$, efficiency of a surfactant $\left(\mathrm{pC}_{20}\right)$, packing parameter $(P)$, and standard free energy interfacial adsorption $\left(\Delta G_{\text {ads }}^{o}\right)$ of SDS in the absence/ presence of $\mathrm{MB}$ were calculated. On the basis of $\Delta G_{\mathrm{m}}^{o}$ values, it was noticed that the increasing volume fraction of methanol disfavors the micellization of SDS, while the presence of MB increased the efficiency of SDS making the micellization process more spontaneous.
\end{abstract}

\section{Introduction}

In the present context, industrialization and urbanization increase the contamination of water, which is a serious problem in human life. Dyes that are used in various industries such as paper, pulp, leather, pharmaceuticals, plastic, paint, rubber, and textiles make a significant contribution to this contamination [1]. They damage humans badly as they cause liver dysfunction and have a negative impact on the brain, central nervous system, kidneys, and reproductive system [2]. The removal of dyes from wastewater is, therefore, very important for the ecosystem and human health. Currently, researchers are doing rapid investigations on new methods for developing efficient and cost-effective technologies for removing environmentally harmful dyes from industrial wastewater. Based on this problem, surfactant micelles are one of the most suitable methods for capturing hydrophobic dyes from wastewater [3]. For the production of many industrial and personal care products such as shower gels, shampoos, and bath additives, concentrated aqueous solutions of anionic surfactants are widely used in a blend with salts and additional additives like dyes and perfumes [4]. In the dye industry, surfactants are frequently used because they can wet and help in the scattering of dyes, particularly which are weakly soluble. The interactions of surfactants with dyes play a crucial role in accomplishing a level of the color [5]. Although much research has been done on dye-surfactant interactions [6-14], studies in this area are still interesting and important for the theory and technology of dyeing [15].

There is a considerable interest in the role of cosolvents for the formation of micelles in surfactant solutions from both fundamental and applied points of view because the use of surfactants in many physicochemical and interface 
phenomena largely depends on the interactional behavior of them [16].

The adding of external additives into the aggregate structure of surfactants will change their physicochemical characteristics such as the degree of ionization, reaction rates, and clouding or phase separation [17-20]. In the same way, the critical micelle concentration of ionic surfactants is significantly altered by the addition of a modest quantity of an organic solvent, since the added organic solvent will, in general, break or make the water structure by solvating the hydrophobic tail of the surfactant by its hydrocarbon (hydrophobic) portion [21]. Huang and Ren [22] studied the effect of isopropanol on the micellization behavior of a binary mixture of amphoteric amino sulfonate surfactant (C12AS) with octylphenol polyoxyethylene ether (10) in water $/ \mathrm{NaCl}$ solution and found that the formation of mixed micelles and the intercalation of C12AS into the mixed micelle is disfavored in the presence of isopropanol. Li et al. [23] studied the effect of short-chain alcohol on the micellization behavior of octadecyltrimethylammonium bromide and found that the presence of alcohol molecule at the air-liquid interface or in the micelle causes the variation in the interfacial or micellization behavior. Ren et al. [24, 25] studied the advantages and disadvantages of isopropanol on the micellization behavior of mixed surfactants based on different thermodynamic parameters which are helpful in designing the surfactant formulas relative to cosolvent.

Methylene blue is a thiazine dye that has an assortment of industrial and scientific applications [26]. The self-association property of methylene blue, a common redox indicator and biological dye, has been studied by several researchers for a long period [27-30].

Sodium dodecyl sulfate is a typical anionic surfactant, and its interactions with chromophores such as ruthenium complexes [31], acridine orange [32], methyl orange, cresol red and methyl red [33], methyl violet [34], methylene blue, C.I. Mordant Black 11, C.I. Mordant Black 17, and C.I. Direct Yellow 50 in aqueous solution [35] have been investigated by researchers.

Several types of interactions between anionic surfactants and cationic dyes are known to occur in solution, including dye-dye dimer formation $[27,29]$, dye-surfactant interaction (including ion-pair formation and dye-surfactant aggregates), [31, 32, 36], and dye-solvent interactions [30, 37]. Many techniques have been used to qualitatively and quantitatively describe dye-surfactant interactions, i.e., potentiometry [38], conductometry [39], ion-selective electrodes [40], and tensiometry [41, 42]. The most frequently used technique to explore dye-surfactant interactions is spectrophotometry $[35,42-46]$.

The properties of the formation of complex ion pairs of SDS-methylene blue have been explored by means of surface tension and UV-Vis/fluorescence measurements [41], molecular spectroscopic measurement [47], photoacoustic imaging technique [13], and UV spectroscopic analysis $[48,49]$ in an aqueous medium, but their interactional properties in alcohol-water mixed solvent media have not yet been examined. We have started to study the interactional properties using lower chain alcohols like methanol, ethanol, and propanol. However, this work mainly focused on the effect of methanol on the interaction between dye and surfactant. To obtain information on the CMC of the individual surfactants and surfactant-dye complex in a binary solvent, the measurements were carried out by tensiometric and conductometric analysis. This study will provide the basic data for designing surfactant formulations in the presence/absence of dye in mixed solvent systems and will be useful to achieve the applications of these formulations for the industrial sector. Also, it provides a further idea to the researchers to carry out the experiment on dye-surfactant interaction in mixed solvent systems.

\section{Experimental}

2.1. Materials. SDS (99\% purity) was purchased from Loba Industries, India. Methylene blue ( $>82 \%$ purity) was purchased from Ranbaxy, India, and used as it was brought. All experiments were carried out with analytical reagent grade chemicals using double-distilled water. Methanol was obtained from Merck, India, with the highest purity (99.9\%) and was used during the experiment. Methanol-water mixtures were prepared in the research laboratory of the Department of Chemistry, Mahendra Morang Adarsh Multiple Campus, Tribhuvan University, Biratnagar, Nepal.

2.2. Measurements. The specific conductance was determined using a digital conductivity meter purchased from Systronics India Ltd. with a dip-type conductivity cell with a cell constant of $1.002 \mathrm{~cm}^{-1}$, which was calibrated with $\mathrm{KCl}$ solution in the appropriate concentration range with an uncertainty of $0.01 \%$. The surface tension was measured using a Kruss Easy Dyne Tensiometer using the du Nouy's ring method. The calibration data of the tensiometer are stored internally, and after base adjustment, the surface tension of pure methanol and water was measured and found to be $22.30 \mathrm{mN} \cdot \mathrm{m}^{-1}$ and $72 \mathrm{mN} \cdot \mathrm{m}^{-1}$, respectively, at $298.15 \mathrm{~K}$ which is in good agreement with the literature [50]. The resulting value from measurements has an uncertainty of $\pm 0.20 \mathrm{mN} \cdot \mathrm{m}^{-1}$. Before each measurement, the ring was first cleaned with distilled water and warmed to a red color with a Bunsen burner. Three successive readings were taken for each concentration of SDS, and their mean value was taken into account. The temperature of all measurements was controlled by a jacketed vessel connected to a thermostated water bath. All measurements were carried out at $298.15 \mathrm{~K}$ within $\pm 0.2 \mathrm{~K}$. SDS was dried for $1 \mathrm{~h}$ below its melting point of $479.15 \mathrm{~K}$ before preparing solutions. The SDS solution having a concentration of $0.1 \mathrm{~mol} \cdot \mathrm{L}^{-1}$ was prepared in the double-distilled water $\left(\gamma=72 \mathrm{mN} \cdot \mathrm{M}^{-1}\right)$, and the measurement of surface tension was carried out at each internal dilution at $298.15 \mathrm{~K}$. The $\mathrm{CMC}$ was determined to be $8.1 \mathrm{mM}$ which is in very close agreement with the literature $[51,52]$. The solution of methylene blue was made in double-distilled water, and absorbance was measured using Labtronics UV Visible Spectrophotometer (Single Beam) at $298.15 \mathrm{~K}$. The peak absorbance was seen at $664 \mathrm{~nm}$ which is in agreement with the literature $[27,47,53,54]$. 


\section{Results and Discussion}

3.1. Conductometric Studies. The CMC values of SDS, calculated from conductivity and surface tension measurements at $298.15 \mathrm{~K}$, are very close to each other and are in close agreement with the literature $[55,56]$. From Figure 1 , it can be seen that the linear region which is above the CMC has a smaller slope than that of the linear region below the CMC. This is the consequence of higher SDS concentrations, which lowers the ionic mobility [10]. It should also be noted that the micelles that are formed at a higher surfactant concentration, far from the CMC, increase the solubility of the dye in solution, which slows down the conductivity [26].

The presence of a cosolvent reduces micelle formation, and it completely inhibits micellization when its concentration reaches an optimum level [57]. The solution of SDS appeared slightly cloudy at 0.4 volume fraction of methanol and resulted in turbidity in the presence of methylene blue, where the distribution of ions/ion pairs was nonuniform. The turbid solution gave fluctuated data of conductivity and surface tension (result not shown), which makes it difficult to locate the CMC and calculate other parameters; therefore, measurements were not carried at/above 0.4 volume fraction of methanol. The CMC increases with the increasing volume fraction of methanol, which is due to the inclusion of methanol in the micelles, where it breaks down the micelles [58]. It is seen that short-chain alcohols increase the CMC at the higher bulk phase concentrations because they lower the cohesive energy density of the water that increases the solubility of the monomeric form of the surfactant [59]. There is an alternative explanation in the case of ionic surfactants that is based on the dielectric constant of the medium. The short-chain alcohols reduce the dielectric constant of the aqueous phase, which increases the mutual repulsion of the ion heads in the micelle, which would reverse micellization and increase the CMC [60].

In addition to the interaction between methylene blue and SDS, interactions between the cosolvent and water, cosolvent and surfactant, and cosolvent and dye also need to be taken into account. Most often, the alcohol hydroxyl group is hydrogen-bonded to the head group of the surfactant and henceforth reduces the attraction between SDS and the dye. Therefore, to explain the inhibitory impact of cosolvents on the development of molecular complexes, we need to consider the sum of several potential variables, such as varying physicochemical properties of the medium, preferential solvation of dye, and cosolvent-water and cosolvent-surfactant interactions. However, it is difficult to choose and decide which factor shows the most inhibitory effects among all. Most often, all of the above variables contribute less or more in different ways [57].

It is seen that both the conductivity and CMC of SDS decrease with the addition of methylene blue because the dye has a positive charge which reduces the mutual repulsion within the anionic moiety of SDS [61] by binding with the micellar aggregates at the surface [34].

We could estimate the number of SDS molecules per micelle, i.e., the aggregation number $(N)$ using relation $N=$ $4 \pi l_{\mathrm{c}}^{2} / A_{\mathrm{m}} \quad[62,63]$, where $l_{\mathrm{c}}=\left[1.54+1.26 n_{\mathrm{c}}\right]$ is a hydrophobic tail length, $A_{\mathrm{m}}$ is the area occupied per surfactant molecule, and $n_{c}$ is the number of carbon atoms in the alkyl chain. Taking $A_{\mathrm{m}}=62.10$ for the SDS from the literature [56], we get $N=56$, which is in agreement with the literature [64] where the value of $N$ was detected to be $55 \pm 5$. Sachin et al. [63] also have reported the $N$ value of SDS to be greater than 50 .

Thus, for ionic surfactant, the standard Gibbs free energy of micellization, $\Delta G_{\mathrm{m}}^{o}$, is evaluated on the basis of the pseudophase separation model [65] by the relation

$$
\Delta G_{\mathrm{m}}^{o}=(2-\alpha) R T \ln X_{\mathrm{CMC}},
$$

where $\alpha$ is the degree of dissociation, which was determined as $\alpha=S_{2} / S_{1}[55,66-68] ; S_{2}$ is the postmicellar slope, $S_{1}$ is the premicellar slope, and $X_{\mathrm{CMC}}$ indicates the mole fraction of CMC. The CMC and $\alpha$ values were calculated by conductometric data on the basis of a conventional procedure called Williams' method [69].

The obtained values of $\Delta G_{\mathrm{m}}^{o}$ are negative, which indicates the spontaneity of the micellization process. This spontaneity decreases with an increasing volume fraction of methanol, after which micellization is less favorable. The $\Delta G_{\mathrm{m}}^{o}$ values of SDS in the absence of dye are in close agreement with the literature [55]. The negative values of $\Delta G_{\mathrm{m}}^{o}$ increase when the dye content is added in mixed systems, which indicates that the formation of micelles becomes more feasible with the addition of the dye.

The values of degree of ionization $(\alpha)$, CMC (by conductometry and tensiometry), and Gibbs free energy of micellization $\left(\Delta G_{\mathrm{m}}^{o}\right)$ of SDS by conductometry with/without methylene blue in different volume fractions of methanolwater at $298.15 \mathrm{~K}$ are tabulated in Table 1.

3.2. Tensiometric Measurements and Surface Properties. More often, the micelle structure of aqueous SDS micelles is in equilibrium with solution monomers and the surfactant monolayer at the air/water interface. The positively charged methylene blue strongly attracts SDS from the equilibrium solution, interacts with it, and forms complexes that have a higher potential to stay at the air/solution interface [41], thereby, lowering the surface tension.

The activity of surfactant in the micelles is lower in the presence of dye, whereby micelles are formed at lower concentrations. It is well understood that the impurity (dye) lowers the surface tension significantly below the CMC and then sufficiently solubilized in micelles or forms a dyesurfactant ion pair after an intermediate minimum (CMC), which leads to a higher surface tension [70]. Thus, a slight increment in the surface tension values is seen after CMC (Figure S1).

The oppositely charged dye-surfactant ion pair acts as a nonionic surfactant and has a greater affinity for the airwater interface, which occupies a larger surface. Thus, the ion pair surfactant has higher efficiency and a lower CMC [71] than the corresponding pure anionic surfactants, as observed in Table 1.

The variation in the slope $(\mathrm{d} \gamma / \mathrm{d} \log C)$ of SDS in the absence/presence of methylene blue with a volume 


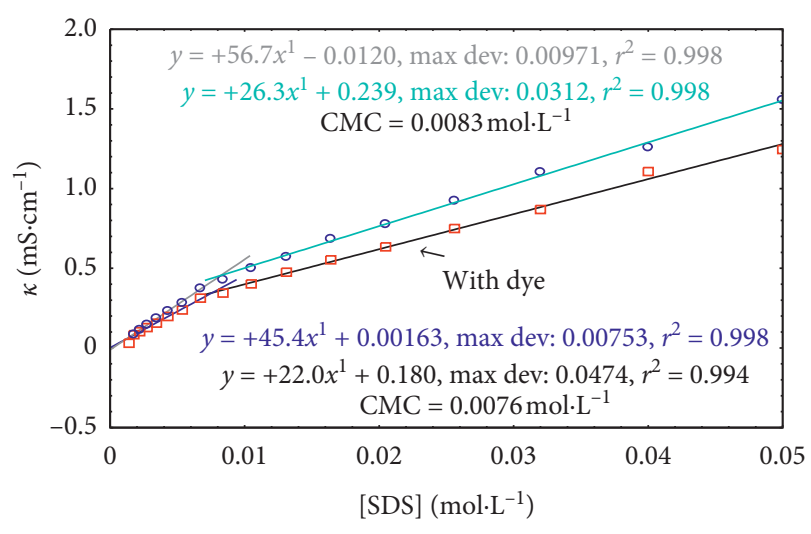

(a)

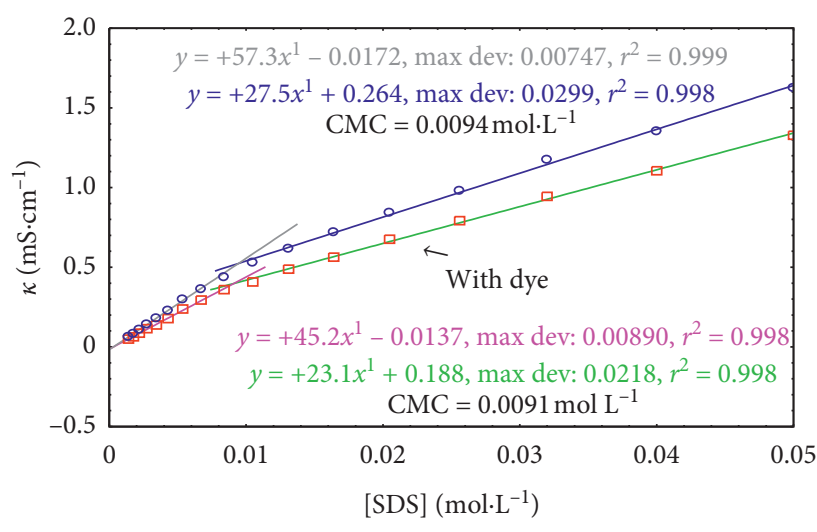

(b)

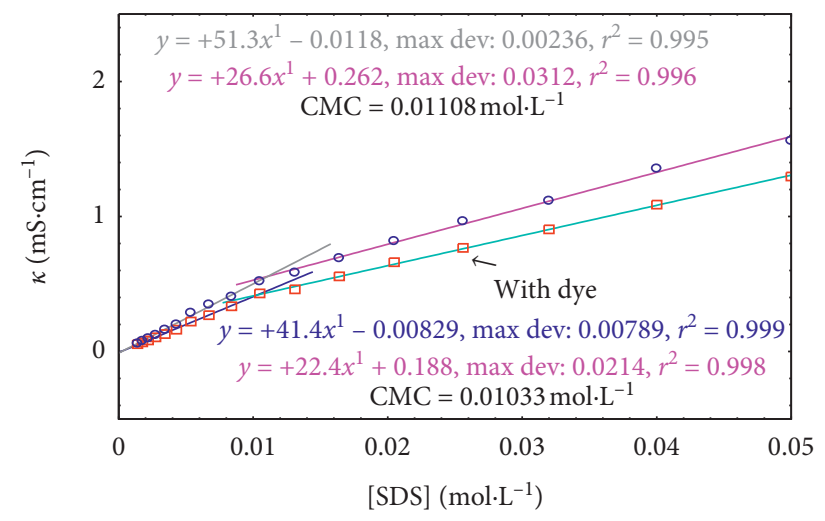

(c)

FIGURE 1: Variation of specific conductance of SDS with concentrations in the absence (o) and presence ( $\square$ ) of methylene blue in (a) 0.1, (b) 0.2 , and (c) 0.3 volume fraction of methanol in aqueous medium at $298.15 \mathrm{~K}$.

fraction of methanol in water is shown in Figure 2. The negative value of the slopes decreases with an increasing volume fraction of methanol, which indicates the lower surface activity of the surfactants. This consequence is due to changes in the dielectric constant and the hydrophobicity of the medium [56]. With the help of slope, the maximum surface excess concentration at the air/methanol-water interface $\Gamma_{\max }$ can be determined by applying Gibb's isotherm [72]:

$$
\Gamma_{\max }=-\frac{1}{2.303 n R T}\left[\frac{\mathrm{d} \gamma}{\operatorname{dlog} C}\right]_{T, P},
$$

where $\gamma$ signifies the surface tension, $R$ is the gas constant $\left(8.314 \mathrm{~J} \mathrm{~mol}^{-1} \cdot \mathrm{K}^{-1}\right), T$ denotes the absolute temperature, $C$ is the surfactant concentration, and $(\mathrm{d} \gamma / \mathrm{d} \log C)$ is the slope of the $\gamma$ versus $\log C$ plot taken at CMC. The constant $n$ is known as a prefactor that takes the value equivalent to 2 for the traditional surfactant, where the surfactant ion and the centerline are univalent.

The area occupied per surfactant molecule $\left(A_{\min }\right)$ at the air/methanol-water interface [73] has been obtained using

$$
A_{\min }=\frac{1}{N_{\mathrm{A}} \Gamma_{\max }}
$$

where $N_{\mathrm{A}}$ is Avogadro's number.
Dissolution of the surfactant molecules leads to the most extreme decrease in surface tension, which is indicated by a parameter $\pi_{\mathrm{CMC}}$ known as surface pressure. $\pi_{\mathrm{CMC}}$ is a measure for the effectiveness of the surfactant to lower the surface tension of the solvent [60] and is calculated using

$$
\pi_{\mathrm{CMC}}=\gamma_{o}-\gamma_{\mathrm{CMC}}
$$

where $\gamma_{o}$ and $\gamma_{\mathrm{CMC}}$ are the values of surface tension of water and the surfactant solution at the CMC, respectively.

According to the data, the surface excess concentration, $\Gamma_{\max }$, decreases with an increasing volume fraction of methanol. This result is due to the lowering of the dielectric constant of the medium and an increase in the hydrophobic effect. Because of these components, the population of surfactant molecules decreases from the surface of the solvent and is dispersed in the bulk [74]. Because of the smaller population of SDS molecules on the superficial or surface region, the minimum surface area, $A_{\text {min }}$, increases and the surface pressure, $\pi_{\mathrm{CMC}}$, decreases with an increasing volume fraction of methanol [56].

The $\Gamma_{\max }$ values of SDS have been found to decrease in the presence of dye. Subsequently, the $A_{\min }$ increases by approximately $11.4 \%, 11.9 \%$, and $29.5 \%$ in the $0.1,0.2$, and 0.3 volume fractions of methanol, respectively, in the presence of dye. This indicates that the dye-surfactant ion 


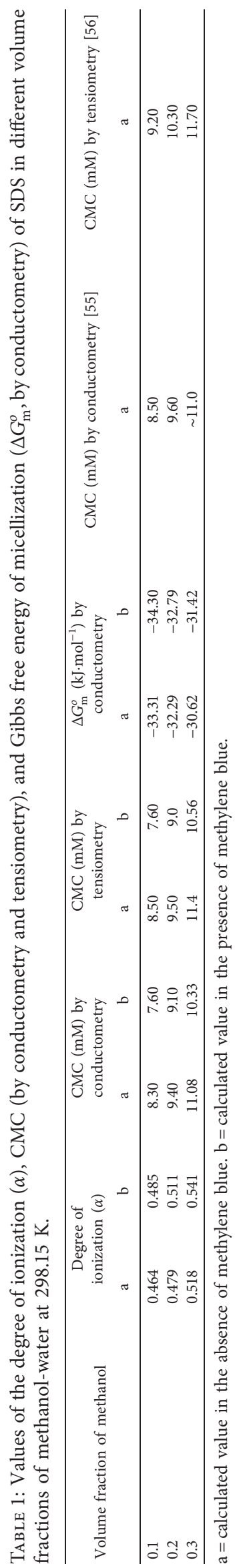




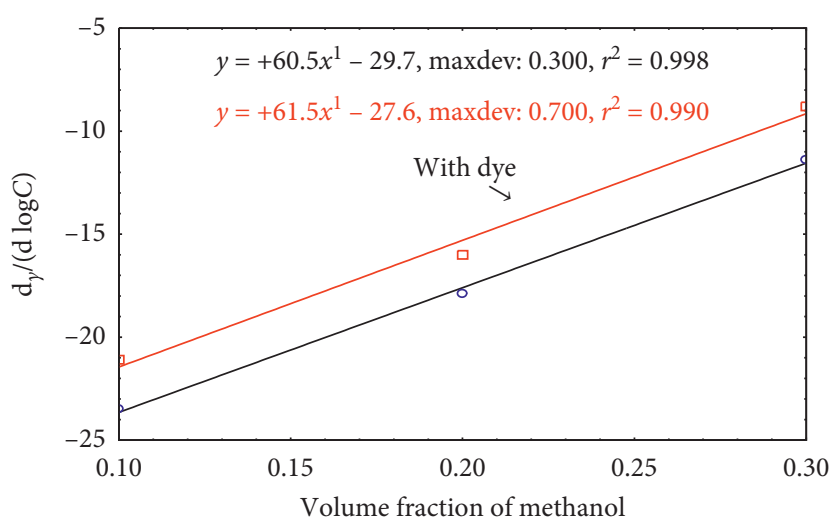

FIGURE 2: Variation in the slope $(\mathrm{d} \gamma / \mathrm{d} \log C)$ of SDS in the absence (o) and presence ( $\square$ ) of methylene blue with different volume fractions of methanol at $298.15 \mathrm{~K}$.

pair occupies more surface area at the air/alcohol-water interface than the surfactant alone. In addition to this, the dye-surfactant ion pair, which is formed by electrostatic interaction, is identical to the new nonionic surfactant with a larger head group, and as a result, a larger surface area per surfactant leads to a lower CMC of the ion-pair surfactant [71]. The values of $\pi_{\mathrm{CMC}}$ increased in the presence of the dye, which suggests that the dye-surfactant ion pair (DSIP) is more effective in lowering the surface tension.

At the air/saturated monolayer interface, the standard free energy interfacial adsorption $\left(\Delta G_{\text {ads }}^{o}\right)$ can be calculated from [75]

$$
\Delta G_{\mathrm{ads}}^{o}=\Delta G_{\mathrm{m}}^{o}-\frac{\pi_{\mathrm{CMC}}}{\Gamma_{\max }}
$$

A higher negative value of $\Delta G_{\mathrm{ads}}^{o}$ indicates a higher efficiency of the surfactant to be adsorbed, that is, spontaneous adsorption at the surface. In this experiment, the $\Delta G_{\text {ads }}^{o}$ values were found to be less negative with an increasing volume fraction of methanol, which indicates low spontaneity of adsorption of surfactant molecules on the surface. However, the values of $\Delta G_{\text {ads }}^{o}$ become more negative in the presence of dye, indicating that the adsorption process is substantially more spontaneous than in the absence of dye.

$\mathrm{pC}_{20}$ is an important parameter for describing the efficiency of a surfactant to reduce the surface tension and is defined in terms of the negative logarithm of $C_{20}$, where $C_{20}$ denotes the concentration of surfactant that is required to reduce the surface tension of the pure solvent by $20 \mathrm{mN} \cdot \mathrm{m}^{-1}$. The value of $\mathrm{pC}_{20}$ has been calculated using $\pi_{\mathrm{CMC}}$ and $\Gamma_{\max }$ [76] from

$$
\mathrm{pC}_{20}=\frac{\pi_{\mathrm{CMC}}-20}{2.303 n R T \Gamma_{\max }}-\log \mathrm{CMC} .
$$

The $\mathrm{pC}_{20}$ values that are calculated from (6) are in good agreement with $-\log C_{20}$ (result not shown), where $C_{20}$ is the practical concentration of surfactant solution whose corresponding surface tension value is reduced by $20 \mathrm{mN} \cdot \mathrm{m}^{-1}$ than that of the methanol-water mixed solvent and can be taken from the plot of Figure S1. Higher adsorption efficiency is indicated by the larger $\mathrm{pC}_{20}$ value, which depends on the hydrophobicity of the medium [67]. On increasing the volume fraction of methanol, the $\mathrm{pC}_{20}$ value decreases; that is, the adsorption at the interface decreases with the increasing population of surfactants in the bulk phase [75]. The efficiency of surfactants is found to be higher with the addition of dye, which is due to the formation of close-packed dye-surfactant ion pairs (DSIP). DSIP behaves like a nonionic surfactant, which usually has higher efficiency than ionic surfactants [60].

According to Israelachvili [77], there is one important parameter called the packing parameter, $P$, which predicts the geometry of the micellar aggregate and can be evaluated by the relation

$$
P=\frac{V_{o}}{A_{\min } l_{c}},
$$

where $V_{o}$ is the volume of exclusion per monomer in the aggregate, as described in Tanford's formula [68, 78]. $V_{o}=\left[27.4+26.9 n_{\mathrm{c}}\right] \AA^{3}, l_{\mathrm{c}}=\left[1.54+1.26 n_{\mathrm{c}}\right] \AA$ is the maximum chain length, and $n_{\mathrm{c}}$ is the number of carbon atoms in the alkyl chain.

The structure of the micelle will be spherical, nonspherical, vesicles, and inverted structures when $P<1 / 3,1 /$ $3<P<1 / 2,1 / 2<P<1$, and $P>1$, respectively. From Table 2 , it can be seen that the values of $P$ are less than $1 / 3$ in both (with/without dye) systems; therefore, the shapes of all the aggregates with/without dye in the mixed solvent are spherical in nature. On increasing the volume fraction of methanol in water, the $P$ values get decreased. This indicates that micellar aggregates get smaller with the addition of methanol [66]. The $P$ values also decrease upon the addition of dye, which might be due to the formation of a dyesurfactant ion pair that has smaller aggregates than the surfactant alone. The $\Gamma_{\max }, A_{\min }, \pi_{\mathrm{CMC}}$, and $P$ values of SDS in the absence of dye are in close agreement with the literature [56].

Table 2 displays the values of $\Delta G_{\mathrm{m}}^{o}, \Gamma_{\max }, A_{\min }, \pi_{\mathrm{CMC}}, P$, $\Delta G_{\text {ads }}^{o}$, and $\mathrm{pC}_{20}$ of SDS in the absence/presence of methylene blue in different volume fractions of methanol at $298.15 \mathrm{~K}$. 


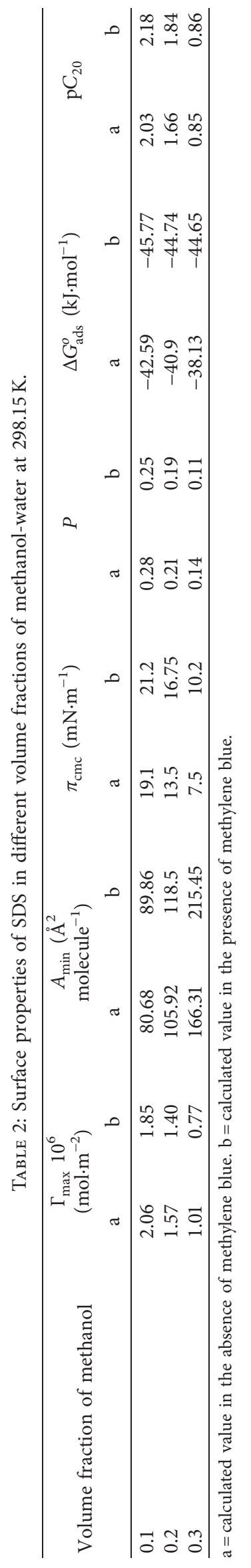


TABLE 3: Various physicochemical parameters of the mixed solvent (methanol-water) at $298.15 \mathrm{~K}$.

\begin{tabular}{lcccc}
\hline $\begin{array}{l}\text { Volume fraction of } \\
\text { methanol }\end{array}$ & $\begin{array}{c}\text { Gordon parameter } \\
\left(\mathrm{G} / \mathrm{J}^{\left.-\mathrm{m}^{-3}\right)}\right.\end{array}$ & $\begin{array}{c}\text { Coefficient of viscosity } \\
\left(\eta_{\mathrm{o}} / \mathrm{mPa} \cdot \mathrm{s}\right)[74]\end{array}$ & $\begin{array}{c}\text { Reichardt's parameter } \\
\left(E_{\mathrm{T}} / \mathrm{kcal} \cdot \mathrm{mol}^{-1}\right)[74]\end{array}$ & $\begin{array}{c}\text { Solvophobic parameter } \\
\left(S_{\mathrm{P}}\right)[79]\end{array}$ \\
\hline 0.1 & 1.91 & 1.0844 & 62.33 & 0.939 \\
0.2 & 1.53 & 1.3106 & 61.56 & 0.862 \\
0.3 & 1.27 & 1.4712 & 60.79 & 0.796 \\
\hline
\end{tabular}

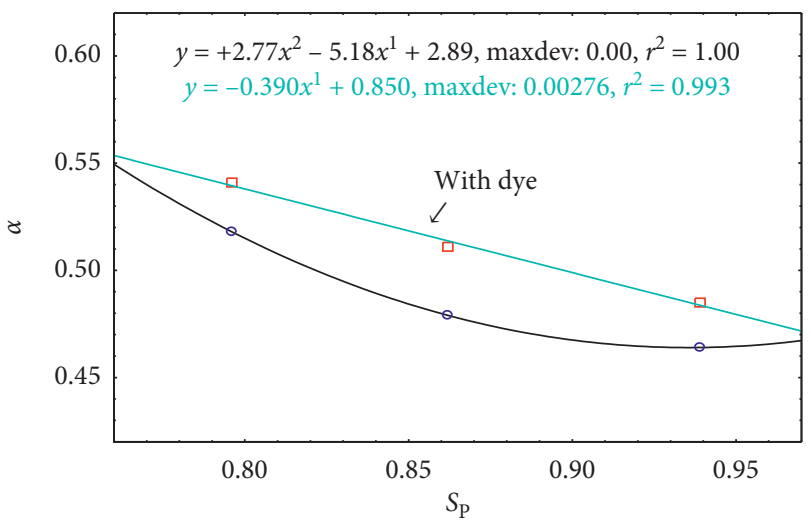

FIgURE 3: Plot of variation of $\alpha$ with solvophobic parameter $\left(S_{\mathrm{P}}\right)$.

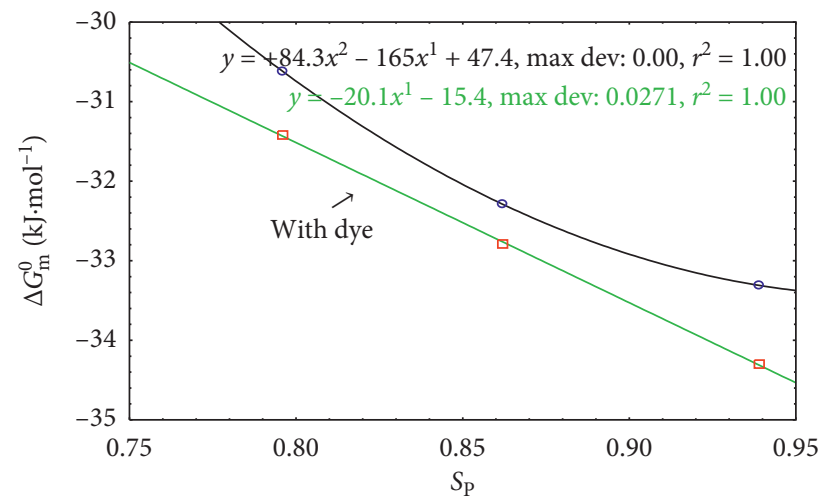

FIGURE 4: Plot of variation of $\Delta G_{\mathrm{m}}^{o}$ with solvophobic parameter $\left(S_{\mathrm{P}}\right)$.

3.3. Correlation of $\Delta G_{\mathrm{m}}^{o}$ with Solvent Parameters. The counterion binding influences $\Delta G_{\mathrm{m}}^{o}$; therefore, $\Delta G_{\mathrm{m}}^{o}$ is the appropriate thermodynamic parameter for ionic surfactants. Here, we correlate $\Delta G_{\mathrm{m}}^{o}$ with different solvent parameters, namely, viscosity, $\eta_{\mathrm{o}}$ (Figure S2a), Gordon parameter, $G$ (Figure S2b), and Reichardt's parameter, $E_{\mathrm{T}}(30)$ (Figure S2c). The $E_{\mathrm{T}}(30)$ and $\eta_{\mathrm{o}}$ values were taken from the literature [74], and $G$ values were calculated by us using [75]

$$
G=\frac{\Upsilon_{\mathrm{sol}}}{V_{\mathrm{m}}^{1 / 3}},
$$

where $\Upsilon_{\text {sol }}$ is the surface tension of the methanol-water mixed solvents and $V_{\mathrm{m}}$ is a molar volume of the mixed solvent.

The $E_{\mathrm{T}}(30)$ values which are depicted in the literature [74] were estimated by the relation

$$
E_{\mathrm{T}}(30)=\frac{h c N_{\mathrm{A}}}{\lambda_{\max }},
$$

where $h$ is a Planck's constant, $c$ is the speed of light, $N_{\mathrm{A}}$ is Avogadro's constant, and $\lambda_{\max }$ is the wavelength of the maximum absorption band in the visible/near-IR region.

All these three parameters $\left(\eta_{\mathrm{o}}, G, E_{\mathrm{T}}\right)$ produced curvilinear correlations with $\Delta G_{\mathrm{m}}^{o}$ in the absence of dye, as obtained by Pan et al. [68], while the above parameters produced linear correlations with $\Delta G_{\mathrm{m}}^{o}$ in the presence of dye. However, further exploration is necessary in this area with different surfactants and dyes in nonaqueous as well as mixed solvents. The development of a database on the solution behavior of surfactants and their interaction with dyes in mixed solvent media, thereby showing quantitative correlations of the results with different solvent parameters, should be a worthwhile research effort in the future. 


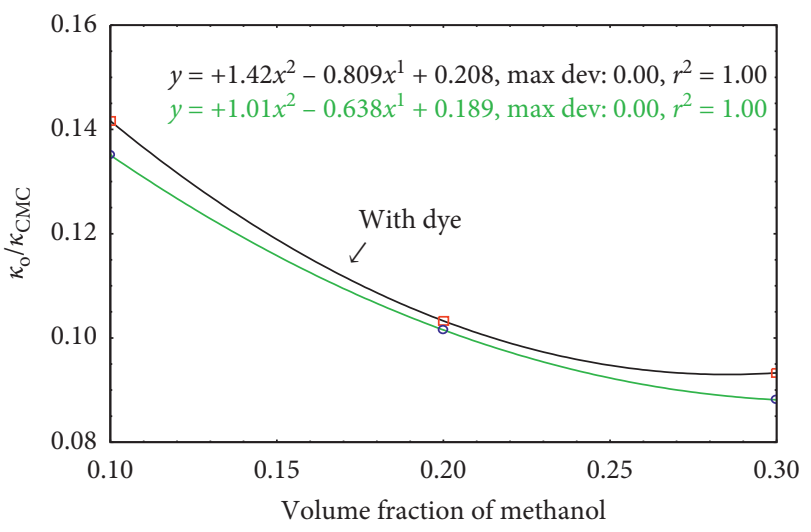

Figure 5: Plot of variation of $\kappa_{o} / \kappa_{\mathrm{CMC}}$ with volume fraction of methanol.

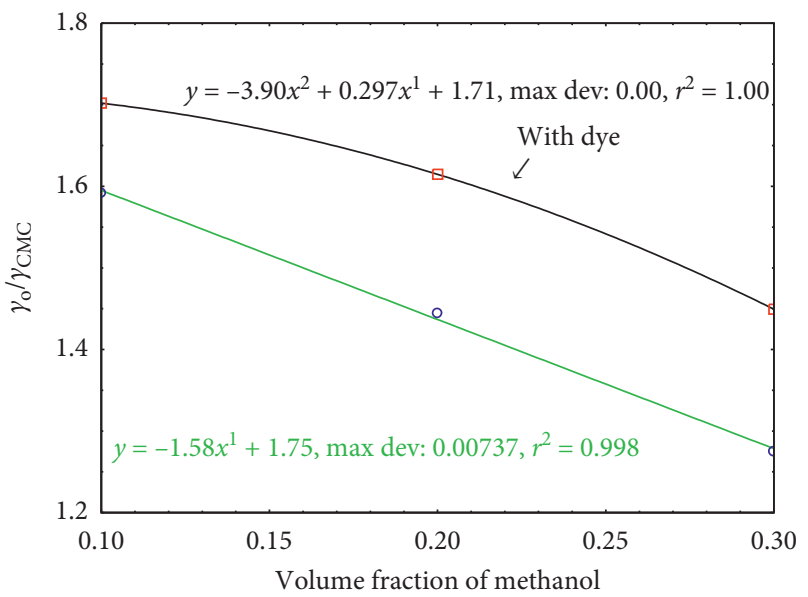

FIGURE 6: Plot of variation of $\gamma_{o} / \gamma_{\mathrm{CMC}}$ with volume fraction of methanol.

3.4. Correlation of $\alpha$ and $\Delta G_{\mathrm{m}}^{o}$ with the Solvophobic Parameter $\left(S_{p}\right)$. The interaction of alkyl chains with solvents was measured by the solvophobic parameters. A smaller $S_{\mathrm{p}}$ value reflects a stronger interaction between the hydrocarbon and solvent. The values of $S_{\mathrm{P}}$ were taken from the literature of Wang et al. [79], which was calculated using (10) mentioned by Abraham et al. [80] as

$$
\begin{aligned}
& S_{\mathrm{p}}=1-\frac{M(\text { solvent })}{M(\text { hexadecane })}, \\
& S_{\mathrm{p}}=1+\frac{M}{4.2024},
\end{aligned}
$$

where $M$ (hexadecane $)=-4.2024, M$ (solvent) is the slope value obtained from the plot of $\Delta G_{\mathrm{t}}^{o}$ versus $R_{\mathrm{T}}$ as given by

$$
\Delta G_{\mathrm{t}}^{o}=M R_{\mathrm{T}}+D,
$$

where $\Delta G_{\mathrm{t}}^{o}$ refers to the transfer of a series of solutes from water to a given solvent, $R_{\mathrm{T}}$ is a solute parameter, and $M$ and $D$ characterize the solvent.

It is seen that the $S_{\mathrm{P}}$ value decreases linearly with an increasing volume fraction of methanol (Table 3 ). This means that the hydrophobic interaction between hydrocarbon and water molecules is maximum at a 0.3 volume fraction of methanol in our study, and this hydrophobic interaction affects the ionization degree of SDS and SDS-MB aggregates in a way that the $\alpha$ values decrease with increasing $S_{\mathrm{P}}$ values (Figure 3 ). The value of $\alpha$ decreases in the curvilinear path in the case of the SDS alone and linearly in the case of the SDS-MB ion pair, respectively. This suggests that the formation of aggregates is favored by increasing the solvophobic power of hydrocarbon chains in a solvent. However, the linear decrease in $\alpha$ with increasing $S_{\mathrm{P}}$ values indicates a higher efficiency of aggregation in the presence of the dye.

Further, from Figure 4, it can be noticed that the $\Delta G_{\mathrm{m}}^{o}$ values of SDS decrease in the curvilinear path with increasing $S_{\mathrm{P}}$ values but decrease linearly in the case of SDS$\mathrm{MB}$ aggregates. The higher negative value of $\Delta G_{\mathrm{m}}^{o}$ at higher $S_{\mathrm{P}}$ values suggests that micellization is more spontaneous at lower interactions between hydrocarbons and solvent. However, micellization is more spontaneous in the presence of the dye than the surfactant alone.

3.5. Correlation of $\kappa_{o} / \kappa_{C M C}$ and $\gamma_{o} / \gamma_{C M C}$ with Volume Fraction of Methanol. Mukhim and Ismail [81] had developed a concept of the ratio of the solvent surface tension to the limiting surface at the $\mathrm{CMC}\left(\gamma_{o} / \gamma_{\mathrm{CMC}}\right)$ to describe the 
solvophobic effect. Sachin et al. [42] also used the same ratio to study the solvent effects. In the same way, the concept of the ratio of the solvent conductance to the conductance at the CMC $\left(\kappa_{o} / \kappa_{\mathrm{CMC}}\right)$ has been developed by our researchers to observe the efficiency of surfactants $[82,83]$. Figure 5 shows the curvilinear variation of $\kappa_{o} / \kappa_{\mathrm{CMC}}$ with volume fraction of methanol. Figure 6 shows the variation of $\gamma_{o} / \gamma_{\text {CMC }}$ with volume fraction of methanol. The plot is linear in the absence of dye and is curvilinear in the presence of dye. The $\kappa_{o} / \kappa_{\mathrm{CMC}}$ and $\gamma_{o} / \gamma_{\mathrm{CMC}}$ value decreases with an increasing volume fraction of methanol, which suggests that the micellization is inhibited by the cosolvent, which might be due to interaction between methanol and methylene blue.

\section{Conclusions}

The CMC of SDS increases, while $\Delta G_{\mathrm{m}}^{o}, \Gamma_{\max }$, and $\pi_{\mathrm{CMC}}$ values decrease, with an increasing volume fraction of methanol in both the absence and presence of the dye. The $\Delta G_{\mathrm{ads}}^{o}$ values become less negative and the $\mathrm{pC}_{20}$ value decreases with an increasing volume fraction of methanol. The $P$ values decrease with an increasing volume fraction of methanol in both the absence and presence of the dye. In the presence of dye, the value of $\Delta G_{\text {ads }}^{o}$ becomes more negative, indicating the spontaneous process of adsorption, and the value of $\pi_{\mathrm{CMC}}$ increases, indicating a higher efficiency of lowering the surface tension. The increase in positive $\mathrm{pC}_{20}$ and negative $\Delta G_{\mathrm{m}}^{o}$ values suggests that the micellization process is more feasible and spontaneous in the presence of dye. The $\Gamma_{\max }$ values of SDS decreased and $A_{\min }$ increased in the presence of dye. The value of the packing parameter, $P$, of all aggregates with/without dye is less than $1 / 3$, indicating that aggregates are spherical in shape.

\section{Data Availability}

The data used in the present study are available from the corresponding authors upon reasonable request.

\section{Conflicts of Interest}

The authors declare that they have no conflicts of interest.

\section{Acknowledgments}

The authors are thankful to the "Ministry of Science and Technology, Kathmandu, Nepal," for providing funds to carry out the experiment in the fiscal year 2074/75 B.S.

\section{Supplementary Materials}

Figure S1: tensiometric profiles of SDS without methylene blue (o) and with methylene blue ( $\square$ ) in (a) 0.1 , (b) 0.2 , and (c) 0.3 volume fraction of methanol in aqueous medium at $298.15 \mathrm{~K}$. Figure S2: plot of variation of $\Delta G_{\mathrm{m}}^{o}$ with (a) viscosity $\left(\eta_{\mathrm{o}}\right)$, (b) Gordon parameter $(G)$, and (c) Reichardt's parameter $\left(E_{\mathrm{T}}(30)\right)$. (Supplementary Materials)

\section{References}

[1] R. Kant, "Textile dyeing industry an environmental hazard," Natural Science, vol. 4, no. 1, p. 22, 2012.

[2] J.-S. Bae and H. S. Freeman, "Aquatic toxicity evaluation of copper-complexed direct dyes to the daphnia magna," Dyes and Pigments, vol. 73, no. 1, p. 126, 2007.

[3] P. Pandit and S. Basu, "Removal of ionic dyes from water by solvent extraction using reverse micelles," Environmental Science \& Technology, vol. 38, no. 8, pp. 2435-2442, 2004.

[4] L. D. Rhein, M. Schlossman, A. O'Lenick, and P. Somasundaran, Surfactants in Personal Care Products and Decorative Cosmetics, CRC Press, Boca Raton, FL, USA, 2006.

[5] Z. A. Khan and A. S. Al-Bogami, "Dye-surfactant interaction: role of solvent," Asian Journal of Chemistry, vol. 25, no. 18, pp. 10499-10503, 2013.

[6] I. M. Imani, N. Noei, and S. Azizian, "Foam analysis of aqueous solution containing ion pair of methyl violet and SDS," Colloids and Surfaces A: Physicochemical and Engineering Aspects, vol. 587, Article ID 124338, 2020.

[7] M. Khamis, B. Bulos, F. Jumean, A. Manassra, and M. Dakiky, "Azo dyes interactions with surfactants. Determination of the critical micelle concentration from acid?base equilibrium," Dyes and Pigments, vol. 66, no. 3, pp. 179-183, 2005.

[8] M. Sarkar and S. Poddar, "Studies on the interaction of surfactants with cationic dye by absorption spectroscopy," Journal of Colloid and Interface Science, vol. 221, no. 2, pp. 181-185, 2000.

[9] A. Srivastava and K. Ismail, "Binding of phenol red to cetylpyridinium chloride at air-solution and micelle-solution interfaces in aqueous ethylene glycol media," Colloids and Surfaces A: Physicochemical and Engineering Aspects, vol. 462, pp. 115-123, 2014.

[10] M. Taj Muhammad and M. N. Khan, "Study of electrolytic effect on the interaction between anionic surfactant and methylene blue using spectrophotometric and conductivity methods," Journal of Molecular Liquids, vol. 234, pp. 309-314, 2017.

[11] A. Tehrani-Bagha and K. Holmberg, "Solubilization of hydrophobic dyes in surfactant solutions," Materials, vol. 6, no. 2, pp. 580-608, 2013.

[12] S. Tul-Muntaha and M. N. Khan, "Conductometric investigation of the interaction of natural and synthetic surfactant with cationic dye in water-alcohol mixed solvent," Journal of Chemical \& Engineering Data, vol. 60, no. 10, pp. 3009-3017, 2015.

[13] J. Wang, C.-Y. Lin, C. Moore, A. Jhunjhunwala, and J. V. Jokerst, "Switchable photoacoustic intensity of methylene blue via sodium dodecyl sulfate micellization," Langmuir, vol. 34, no. 1, pp. 359-365, 2018.

[14] A. Zdziennicka and B. Jańczuk, "Behavior of anionic surfactants and short chain alcohols mixtures in the monolayer at the water-air interface," Journal of Surfactants and Detergents, vol. 14, no. 2, pp. 257-267, 2011.

[15] B. Simoncic, "A study of anionic dye-cationic surfactant interactions in mixtures of cationic and nonionic surfactants," Dyes and Pigments, vol. 54, no. 3, pp. 221-237, 2002.

[16] L. L. Schramm, E. N. Stasiuk, and D. G. Marangoni, "2 surfactants and their applications," Annual Reports Section, vol. 99, pp. 3-48, 2003.

[17] D. Kumar and M. A. Rub, "Studies of interaction between ninhydrin and Gly-Leu dipeptide: influence of cationic surfactants (m-s-m type Gemini)," Journal of Molecular Liquids, vol. 269, pp. 1-7, 2018. 
[18] D. Kumar and M. A. Rub, "Catalytic role of 16-s-16 micelles on condensation reaction of ninhydrin and metal-dipeptide complex," Journal of Physical Organic Chemistry, vol. 32, no. 4, p. e3918, 2018.

[19] D. Kumar and M. A. Rub, "Role of cetyltrimethylammonium bromide (CTAB) surfactant micelles on kinetics of [Zn(II)Gly-Leu]+ and ninhydrin," Journal of Molecular Liquids, vol. 274, pp. 639-645, 2019.

[20] S. Mahbub, M. Abdul Rub, and M. A. Hoque, "Clouding and thermodynamic characteristics of triton X-100 in the presence of ciprofloxacin hydrochloride: influence of polyols," Journal of Chemical \& Engineering Data, vol. 64, no. 9, pp. 4181-4188, 2019.

[21] R. Palepu, H. Gharibi, D. M. Bloor, and E. Wyn-Jones, "Electrochemical studies associated with the micellization of cationic surfactants in aqueous mixtures of ethylene glycol and glycerol," Langmuir, vol. 9, no. 1, pp. 110-112, 1993.

[22] J. Huang and Z. H. Ren, "Micellization of binary mixture of amino sulfonate amphoteric surfactant with octylphenol polyoxyethylene ether (10) in water/ $\mathrm{NaCl}$ solution: effect of isopropanol," Journal of the Iranian Chemical Society, vol. 16, no. 6, pp. 1345-1353, 2019.

[23] D. M. Li, J. Huang, Z. H. Ren et al., "Interfacial properties and micellization of octadecyltrimethylammonium bromide in aqueous solution containing short chain alcohol and effect of chain length of alcohol," Journal of Dispersion Science and Technology, vol. 41, no. 6, pp. 856-862, 2020.

[24] Z. H. Ren, J. Huang, Y. Luo et al., "Effect of isopropanol on the micellization of binary mixture containing amino sulfonate amphoteric surfactant in aqueous solution: mixing with sodium dodecylbenzene sulfonate," Journal of the Taiwan Institute of Chemical Engineers, vol. 65, pp. 482-487, 2016.

[25] Z. H. Ren, J. Huang, Y. Luo et al., "Effect of isopropanol on the micellization of binary mixtures containing amino sulfonate amphoteric surfactant in aqueous solution: mixing with octadecyltrimethyl ammonium bromide," Colloids and Surfaces A: Physicochemical and Engineering Aspects, vol. 504, pp. 131-138, 2016.

[26] A. G. Gilani, H. Tajalli, and M. S. Zakerhamidi, "Photophysical behavior of thiazine dyes with or without surfactants into poly-HEMA hydrophilic gel matrix," Journal of Molecular Liquids, vol. 143, no. 2-3, pp. 81-88, 2008.

[27] K. Bergmann and C. T. O'Konski, "A spectroscopic study OF methylene blue monomer, dimer, and complexes with montmorillonite," The Journal of Physical Chemistry, vol. 67, no. 10, pp. 2169-2177, 1963.

[28] A. Katafias, P. Kita, G. Wrzeszcz, and A. Mills, "Kinetics of the methylene blue oxidation by cerium(IV) in sulphuric acid solutions," Transition Metal Chemistry, vol. 32, no. 1, pp. 31-37, 2007.

[29] P. Mukerjee and A. K. Ghosh, "“Isoextraction" method and the study of the self-association of methylene blue in aqueous solutions," Journal of the American Chemical Society, vol. 92, no. 22, pp. 6403-6407, 1970.

[30] E. Rabinowitch and L. F. Epstein, "Polymerization of dyestuffs in solution. Thionine and methylene blue," Journal of the American Chemical Society, vol. 63, no. 1, pp. 69-78, 1941.

[31] J. H. Baxendale and M. A. J. Rodgers, "Fluorescence of tris(2,2'-bipyridyl)ruthenium(II) in sodium dodecyl sulfate solutions below the critical micelle concentration," The Journal of Physical Chemistry, vol. 86, no. 25, pp. 4906-4909, 1982.
[32] S. H. Chou and M. J. Wirth, "Rotational diffusion of Acridine Orange attached to SDS micelles," The Journal of Physical Chemistry, vol. 93, no. 22, pp. 7694-7698, 1989.

[33] F. Ahmadi, M. A. Daneshmehr, and M. Rahimi, "The effect of anionic and cationic surfactants on indicators and measurement of dissociation constants with two different methods," Spectrochimica Acta Part A: Molecular and Biomolecular Spectroscopy, vol. 67, no. 2, pp. 412-419, 2007.

[34] K. Edbey, N. Bader, F. Eltaboni, A. Elabidi, S. Albaba, and M. Ahmed, "Conductometric and spectrophotometric study of the interaction of methyl violet with sodium dodecyl sulfate," International Research Journal of Pure and Applied Chemistry, vol. 9, no. 4, pp. 1-7, 2015.

[35] M. Bielska, A. Sobczyńska, and K. Prochaska, "Dye-surfactant interaction in aqueous solutions," Dyes and Pigments, vol. 80, no. 2, pp. 201-205, 2009.

[36] S. J. Atherton and C. M. G. Dymond, "Formation of clusters between ionic species and sodium dodecyl sulfate below the critical micelle concentration: ethidium ions and divalent metal ions," The Journal of Physical Chemistry, vol. 93, no. 18, pp. 6809-6813, 1989.

[37] T. G. Dewey, P. S. Wilson, and D. H. Turner, "Solvent effects on stacking. A kinetic and spectroscopic study of thionine association in aqueous alcohol solutions," Journal of the American Chemical Society, vol. 100, no. 14, pp. 4550-4554, 1978.

[38] B. Simoncic and J. Span, "A study of dye-surfactant interactions. Part 1. Effect of chemical structure of acid dyes and surfactants on the complex formation," Dyes Pigments, vol. 36, no. 1, pp. 1-14, 1998.

[39] S. Bračko and J. Špan, "Conductometric investigation of dye-surfactant ion pair formation in aqueous solution," Dyes Pigments, vol. 45, pp. 97-102, 2000.

[40] S. M. Ghoreishi, M. Behpour, and M. Shabani-Nooshabadi, "Interaction of anionic azo dye and TTAB: cationic surfactant," Journal of the Brazilian Chemical Society, vol. 20, no. 3 , pp. $460-465,2009$.

[41] H. C. Junqueira, D. Severino, L. G. Dias, M. S. Gugliotti, and M. S. Baptista, "Modulation of methylene blue photochemical properties based on adsorption at aqueous micelle interfaces," Physical Chemistry Chemical Physics, vol. 4, no. 11, pp. 2320-2328, 2002.

[42] K. M. Sachin, S. A. Karpe, M. Singh, and A. Bhattarai, "Selfassembly of sodium dodecylsulfate and dodecyltrimethylammonium bromide mixed surfactants with dyes in aqueous mixtures," Royal Society Open Science, vol. 6, no. 3, Article ID 181979, 2019.

[43] P. K. Behera, S. Mohapatra, S. Patel, and B. K. Mishra, "Dyesurfactant interaction: solubilization of styryl pyridinium dyes of varying alkyl chain in alfa-olefinic sulfonate and linear alkyl benzene sulfonate solutions," Journal of Photochemistry and Photobiology A: Chemistry, vol. 169, no. 3, pp. 253-260, 2005.

[44] M. H. Gehlen, M. Ferreira, and M. G. Neumann, "Interaction of methyl orange with cationic micelles and its effect on dye photochemistry," Journal of Photochemistry and Photobiology A: Chemistry, vol. 87, no. 1, pp. 55-60, 1995.

[45] A. K. Jana and S. Rajavenii, "Studies on the molecular interaction of phenazine dyes with Triton X-100," Spectrochimica Acta Part A: Molecular and Biomolecular Spectroscopy, vol. 60, no. 8-9, pp. 2093-2097, 2004.

[46] Ç. Kartal and H. Akbaş, "Study on the interaction of anionic dye-nonionic surfactants in a mixture of anionic and nonionic surfactants by absorption spectroscopy," Dyes and Pigments, vol. 65, no. 3, pp. 191-195, 2005. 
[47] M. K. Carroll, M. A. Unger, A. M. Leach, M. J. Morris, C. M. Ingersoll, and F. V. Bright, "Interactions between methylene blue and sodium dodecyl sulfate in aqueous solution studied by molecular spectroscopy," Applied Spectroscopy, vol. 53, no. 7, pp. 780-784, 1999.

[48] J.-H. Huang, C.-F. Zhou, G.-M. Zeng et al., "Studies on the solubilization of aqueous methylene blue in surfactant using MEUF," Separation and Purification Technology, vol. 98, pp. 497-502, 2012.

[49] S. Yamamoto, S. Kobashi, K.-I. Tsutsui, and Y. Sueishi, "Spectroscopic studies of the interaction between methylene blue-naphthol orange complex and anionic and cationic surfactants," Spectrochimica Acta Part A: Molecular and Biomolecular Spectroscopy, vol. 66, no. 2, pp. 302-306, 2007.

[50] L. Cheng, D. Mewes, and A. Luke, "Boiling phenomena with surfactants and polymeric additives: a state-of-the-art review," International Journal of Heat and Mass Transfer, vol. 50, no. 13-14, pp. 2744-2771, 2007.

[51] X. Zhang, J. K. Jackson, and H. M. Burt, "Determination of surfactant critical micelle concentration by a novel fluorescence depolarization technique," Journal of Biochemical and Biophysical Methods, vol. 31, no. 3-4, pp. 145-150, 1996.

[52] S. A. Markarian, L. R. Harutyunyan, and R. S. Harutyunyan, "The properties of mixtures of sodium dodecylsulfate and diethylsulfoxide in water," Journal of Solution Chemistry, vol. 34, no. 3, pp. 361-368, 2005.

[53] T. Handa, C. Ichihashi, I. Yamamoto, and M. Nakagaki, "The location and microenvironment of dimerizing cationic dyes in lipid membranes as studied by means of their absorption spectra," Bulletin of the Chemical Society of Japan, vol. 56, no. 9, pp. 2548-2554, 1983.

[54] L. M. Moreira, J. P. Lyon, A. Lima et al., "The methylene blue self-aggregation in water/organic solvent mixtures: relationship between solvatochromic properties and singlet oxygen production," Orbital-The Electronic Journal of Chemistry, vol. 9, no. 4, pp. 279-289, 2017.

[55] T. P. Niraula, S. K. Chatterjee, and A. Bhattarai, "Micellization of sodium dodecyl sulphate in presence and absence of alkali metal halides at different temperatures in water and methanol-water mixtures," Journal of Molecular Liquids, vol. 250, pp. 287-294, 2018.

[56] T. P. Niraula, S. K. Shah, S. K. Chatterjee, and A. Bhattarai, "Effect of methanol on the surface tension and viscosity of sodiumdodecyl sulfate (SDS) in aqueous medium at $298.15-323.15^{\circ} \mathrm{K}$," Karbala International Journal of Modern Science, vol. 4, no. 1, pp. 26-34, 2018.

[57] S. Gokturk and M. Tuncay, "Dye-surfactant interaction in the premicellar region," Journal of Surfactants and Detergents, vol. 6, no. 4, pp. 325-330, 2003.

[58] H. N. Singh and S. Swarup, "Effect of monohydroxy alcohols and urea on the CMC of surfactants," Bulletin of the Chemical Society of Japan, vol. 51, no. 5, pp. 1534-1538, 1978.

[59] M. J. Schick and A. H. Gilbert, "Effect of urea, guanidinium chloride, and dioxane on the C.M.C. of branched-chain nonionic detergents," Journal of Colloid Science, vol. 20, no. 5, pp. 464-472, 1965.

[60] M. J. Rosen, Surfactants and Interfacial Phenomena, John Wiley \& Sons, Hoboken, NJ, USA, 2004.

[61] I. Benito, M. A. García, C. Monge, J. M. Saz, and M. L. Marina, "Spectrophotometric and conductimetric determination of the critical micellar concentration of sodium dodecyl sulfate and cetyltrimethylammonium bromide micellar systems modified by alcohols and salts," Colloids and Surfaces A:
Physicochemical and Engineering Aspects, vol. 125, no. 2-3, pp. 221-224, 1997.

[62] M. N. Wadekar, J. Boekhoven, W. F. Jager, G. J. M. Koper, and S. J. Picken, "Micellization behavior of aromatic moiety bearing hybrid fluorocarbon sulfonate surfactants," Langmuir, vol. 28, no. 7, pp. 3397-3402, 2012.

[63] K. M. Sachin, S. A. Karpe, M. Singh, and A. Bhattarai, "Study on surface properties of sodiumdodecyl sulfate and dodecyltrimethylammonium bromide mixed surfactants and their interaction with dyes," Heliyon, vol. 5, no. 4, Article ID e01510, 2019.

[64] N. J. Turro and A. Yekta, "Luminescent probes for detergent solutions. A simple procedure for determination of the mean aggregation number of micelles," Journal of the American Chemical Society, vol. 100, no. 18, pp. 5951-5952, 1978.

[65] C. Carnero Ruiz, "Thermodynamics of micellization of tetradecyltrimethylammonium bromide in ethylene glycol-water binary mixtures," Colloid \& Polymer Science, vol. 277, no. 7, pp. 701-707, 1999.

[66] S. K. Shah, S. K. Chatterjee, and A. Bhattarai, "The effect of methanol on the micellar properties of dodecyltrimethylammonium bromide (DTAB) in aqueous medium at different temperatures," Journal of Surfactants and Detergents, vol. 19, no. 1, pp. 201-207, 2016.

[67] R. Sharma, A. Kamal, and R. K. Mahajan, "A quantitative appraisal of the binding interactions between an anionic dye, alizarin red S, and alkyloxypyridinium surfactants: a detailed micellization, spectroscopic and electrochemical study," Soft Matter, vol. 12, no. 6, pp. 1736-1749, 2016.

[68] A. Pan, B. Naskar, G. K. S. Prameela et al., "Amphiphile behavior in mixed solvent media I: self-aggregation and ion association of sodium dodecylsulfate in 1,4-dioxane-water and methanol-water media," Langmuir, vol. 28, no. 39, pp. 13830-13843, 2012.

[69] J. Goronja, A. Janosevic-Lezaic, B. Dimitrijevic, A. Malenovic, D. Stanisavljev, and N. Pejic, "Determination of critical micelle concentration of cetyltrimethylammonium bromide: different procedures for analysis of experimental data," Chemical Industry, vol. 70, no. 4, pp. 485-492, 2016.

[70] P. Mukerjee and K. Mysels, Critical Micelle Concentrations of Aqueous Surfactant Systems, John Wiley \& Sons, Hoboken, NJ, USA, 1971.

[71] B. Gohain and R. K. Dutta, "Premicellar and micelle formation behavior of dye surfactant ion pairs in aqueous solutions: deprotonation of dye in ion pair micelles," Journal of Colloid and Interface Science, vol. 323, no. 2, pp. 395-402, 2008.

[72] I. Mukherjee, S. P. Moulik, and A. K. Rakshit, “Tensiometric determination of Gibbs surface excess and micelle point: a critical revisit," Journal of Colloid and Interface Science, vol. 394, pp. 329-336, 2013.

[73] G. Sugihara, A. Miyazono, S. Nagadome, T. Oida, Y. Hayashi, and J.-S. Ko, "Adsorption and micelle formation of mixed surfactant systems in water II: a combination of cationic gemini-type surfactant with MEGA-10," Journal of Oleo Science, vol. 52, no. 9, pp. 449-461, 2003.

[74] S. K. Shah, S. K. Chatterjee, and A. Bhattarai, "Micellization of cationic surfactants in alcohol-water mixed solvent media," Journal of Molecular Liquids, vol. 222, pp. 906-914, 2016.

[75] S. Das, S. Mondal, and S. Ghosh, "Physicochemical studies on the micellization of cationic, anionic, and nonionic surfactants in water-polar organic solvent mixtures," Journal of Chemical \& Engineering Data, vol. 58, no. 9, pp. 2586-2595, 2013. 
[76] C. Zhang, T. Geng, Y. Jiang, L. Zhao, H. Ju, and Y. Wang, "Impact of $\mathrm{NaCl}$ concentration on equilibrium and dynamic surface adsorption of cationic surfactants in aqueous solution," Journal of Molecular Liquids, vol. 238, pp. 423-429, 2017.

[77] J. N. Israelachvili, D. J. Mitchell, and B. W. Ninham, "Theory of self-assembly of hydrocarbon amphiphiles into micelles and bilayers," Journal of the Chemical Society, Faraday Transactions 2, vol. 72, pp. 1525-1568, 1976.

[78] C. C. Ruiz, L. Díaz-López, and J. Aguiar, "Micellization of sodium dodecyl sulfate in glycerol aqueous mixtures," Journal of Dispersion Science and Technology, vol. 29, no. 2, pp. 266-273, 2008.

[79] J. Wang, L. Zhang, H. Wang, and C. Wu, “Aggregation behavior modulation of 1-dodecyl-3-methylimidazolium bromide by organic solvents in aqueous solution," The Journal of Physical Chemistry B, vol. 115, no. 17, pp. 4955-4962, 2011.

[80] M. H. Abraham, P. L. Grellier, and R. A. McGill, "A quantitative measure of solvent solvophobic effect," Journal of the Chemical Society, Perkin Transactions, vol. 2, no. 3, p. 339, 1988.

[81] T. Mukhim and K. Ismail, "Aggregation, counter ion binding and adsorption behaviors of cetylpyridinium chloride in water/glycerol media at $25^{\circ} \mathrm{C}$," Journal of Surfactants and Detergents, vol. 15, no. 1, pp. 47-51, 2012.

[82] N. K. Chaudhary, A. Bhattarai, B. Guragain, and A. Bhattarai, "Conductivity, surface tension, and comparative antibacterial efficacy study of different brands of soaps of Nepal," Journal of Chemistry, vol. 2020, Article ID 6989312, 13 pages, 2020.

[83] A. Bhattarai, "Micellization behavior of cetyltrimethylammonium bromide in the absence and presence of sodium polystyrene sulfonate in water and methanol-water mixture: a conductivity approach," Journal of Molecular Liquids, vol. 292, Article ID 111352, 2019. 